\title{
Cisplatin/Fluorouracil/Trastuzumab Regimen
}

National Cancer Institute

\section{Source}

National Cancer Institute. Cisplatin/Fluorouracil/Trastuzumab Regimen. NCI Thesaurus.

Code C141505.

A regimen consisting of cisplatin, fluorouracil and trastuzumab that can be used in the treatment of gastric cancer. 